\title{
Interaction of yeast RNA-binding proteins Nrd1 and Nab3 with RNA polymerase II terminator elements
}

\author{
KRISTINA L. CARROLL, ${ }^{1}$ RODOLFO GHIRLANDO, ${ }^{2}$ JESSICA M. AMES, ${ }^{1}$ and JEFFRY L. CORDEN ${ }^{1}$ \\ ${ }^{1}$ Department of Molecular Biology and Genetics, Johns Hopkins University School of Medicine, Baltimore, Maryland 21205, USA \\ ${ }^{2}$ Laboratory of Molecular Biology, National Institute of Diabetes and Digestive and Kidney Diseases, National Institutes of Health, \\ Bethesda, Maryland 20892-0540, USA
}

\begin{abstract}
Yeast RNA-binding proteins Nrd1 and Nab3 direct transcription termination of sn/snoRNA transcripts, some mRNA transcripts, and a class of intergenic and anti-sense transcripts. Recognition of Nrd1- and Nab3-binding sites is a critical first step in the termination and subsequent processing or degradation of these transcripts. In this article, we describe the purification and characterization of an Nrd1-Nab3 heterodimer. This Nrd1-Nab3 complex binds specifically to RNA sequences derived from a snoRNA terminator. The relative binding to mutant terminators correlates with the in vivo termination efficiency of these mutations, indicating that the primary specificity determinant in nonpoly(A) termination is Nrd1-Nab3 binding. In addition, several snoRNA terminators contain multiple Nrd1- and Nab3-binding sites and we show that multiple heterodimers bind cooperatively to one of these terminators in vitro.
\end{abstract}

Keywords: snoRNA; RNA-binding domain; transcription termination

\section{INTRODUCTION}

The transcriptome of a eukaryotic cell is determined not only by where transcription initiates but also by where it stops and how the primary transcript thus generated is processed. While transcription factors and chromatin proteins play important roles, primarily at the initiation stage, RNA-binding proteins are critical in many of the subsequent steps in synthesis and processing of eukaryotic transcripts. RNA-binding proteins, particularly those containing an RNA-recognition motif (RRM) (Maris et al. 2005), play multiple roles in RNA polymerase II (Pol II) transcription, including capping, splicing, and formation of the transcript 3' end (Bentley 2005; Moore 2005).

Two pathways for Pol II $3^{\prime}$ end formation have been described in yeast. One leads to formation of mRNAs with stable poly(A) tails (Zhao et al. 1999; Proudfoot and O'Sullivan 2002) while the other leads to formation of nonpoly(A) RNAs like small nuclear RNAs (snRNAs) and small nucleolar RNAs (snoRNAs) (Steinmetz et al. 2001). One common feature of both pathways is that signal

\footnotetext{
Reprint requests to: Jeffry L. Corden, Department of Molecular Biology and Genetics, Johns Hopkins University School of Medicine, 725 N. Wolfe Street, Baltimore, MD 21205, USA; e-mail: jcorden@jhmi.edu; fax: (410) 502-6718.

Article published online ahead of print. Article and publication date are at http://www.rnajournal.org/cgi/doi/10.1261/rna.338407.
}

sequences in the nascent transcript bind protein complexes that direct both transcription termination and processing of the transcript.

Termination of snRNAs and snoRNAs requires the RNA-binding proteins Nrd1 and Nab3, the RNA helicase Sen1, and the Pol II C-terminal domain (CTD) (Steinmetz et al. 2001). In addition, Nrd1 and Nab3 form a complex with the nuclear exosome (Vasiljeva and Buratowski 2006), a collection of RNases that is required for degradation of messages or exonucleolytic trimming of the $3^{\prime}$ end of snoRNA precursors (Mitchell et al. 1997; Butler 2002; Houseley et al. 2006). The Nrd1-Nab3 pathway has also been shown to be involved in regulation of Nrd1 levels through premature transcription termination (Arigo et al. 2006a; Houalla et al. 2006) and in termination of cryptic unstable transcripts (CUTS) (Arigo et al. 2006b; LykkeAndersen and Jensen 2006; Thiebaut et al. 2006). In each of these cases, recognition of Nrd1- and Nab3-binding sites is an important first step in termination and subsequent degradation or processing of the Pol II transcript.

What are the signals that direct termination and exosome trimming/degradation of nonpoly(A) transcripts? Mutational screens have identified the sequence motifs GUA[A/G] and UCUU as important elements of nonpoly(A) terminators (Steinmetz et al. 2001; Morlando et al. 2002; Carroll et al. 2004; Steinmetz et al. 2006), and the single RRM of Nrd1 and Nab3, respectively, have been 
shown to bind these sequences (Steinmetz and Brow 1996, 1998; Carroll et al. 2004). Such sequences are preferentially located downstream of snRNA and snoRNA genes (Carroll et al. 2004) although their relative orientation and spacing are not highly conserved.

In this article we describe the coexpression and purification of yeast Nrd1 and Nab3 in Escherichia coli. These proteins form a heterodimer that contains two RRM domains. We show here that the Nrd1-Nab3 complex binds specifically to RNA sequences identified as in vivo nonpoly $(A)$ terminators. The relative binding to mutant terminators mirrors the in vivo effect of these mutations, indicating that $\mathrm{Nrd1}$ and $\mathrm{Nab3}$ binding is the primary specificity determinant in nonpoly $(A)$ termination. Several snoRNA terminators contain multiple potential Nrd1-Nab3binding sites and we demonstrate that multiple heterodimers bind with apparent cooperativity to one of these terminators in vitro. Nrd1-Nab3 RNA binding is discussed in terms of the possible role this interaction plays in termination.

\section{RESULTS}

\section{Expression and purification of Nrd1-Nab3 heterodimer complexes}

Previous work established genetic and physical interactions between two yeast RNA-binding proteins, Nrd1 and Nab3 (Conrad et al. 2000). We coexpressed and purified these proteins in E. coli (see Materials and Methods) to further study their interaction with RNA. The maps shown in Figure 1A indicate the regions of Nrd1 and Nab3 that yield abundant stable protein when coexpressed in bacteria. The expression vector includes all Nrd1 sequences except that encoding the C-terminal 26 amino acids. The deleted region contains a stretch of eight consecutive glutamine residues that are proteolytically cleaved when expressed in E. coli. For stable expression of Nab3, we removed 191 amino acids from the $\mathrm{N}$-terminus and 238 amino acids from the C-terminus. In each case, these regions contain long stretches of simple sequences that are unstable when expressed in E. coli. The N-terminal Nab3 sequence is over 50\% Asp/Glu, with one run of 37 consecutive acidic residues, while the $\mathrm{C}$-terminal domain of Nab3 is $26 \%$ Gln. The N-terminal deletion is viable in yeast whereas the C-terminal deletion is not. However, the 375 amino acid central core of Nab3 retains all of the sequences that are conserved among different organisms.
Thus, all of the conserved motifs, which include single RRMs in both Nrd1 and Nab3, as well as the CTDinteraction domain (CID) of Nrd1 are contained in the bacterially expressed heterodimer.

Coexpressed Nrd1 and Nab3 copurified over several columns (see Materials and Methods), indicating the formation of a tight complex (Fig. 1B). To determine the stoichiometry of the Nrd1-Nab3 complex, sedimentation equilibrium experiments on Nrd1-Nab3 were carried out at rotor speeds of 6-14 krpm and loading concentrations of 3.1, 6.1, and 9.2 $\mu \mathrm{M}$. A global data analysis in terms of a single ideal solute returned excellent fits with a molecular mass of $102 \pm 6 \mathrm{kDa}$ (Table 1; data not shown) that is consistent with a 1:1 Nrd1:Nab3 stoichiometry. Data for each loading concentration were similarly analyzed in terms of a single ideal solute only to obtain identical molecular masses. There is no evidence for dissociation of the heterodimer, and the absence of an increase in the molecular mass with increasing Nrd1-Nab3 concentration indicates that the heterodimers do not interact to form tetramers. The monodispersity of the sample was confirmed by sedimentation velocity experiments, which return a sedimentation coefficient $\left(s_{20, \mathrm{w}}\right)$ of $4.3 \pm 0.1 \mathrm{~S}$ at a loading concentration of $10 \mu \mathrm{M}$ (Fig. 6A, below; Table 1). At $21.5 \mu \mathrm{M}$ the Nrd1-Nab3 complex is also monodisperse, having a measured $s_{20, \mathrm{w}}$ of $4.2 \pm 0.1 \mathrm{~S}$ (data not shown). This value further confirms the lack of any Nrd1Nab3 self-association at the concentrations studied.

The frictional ratio $\left(f / f_{o}\right)$ compares the observed frictional coefficient with the frictional coefficient expected for

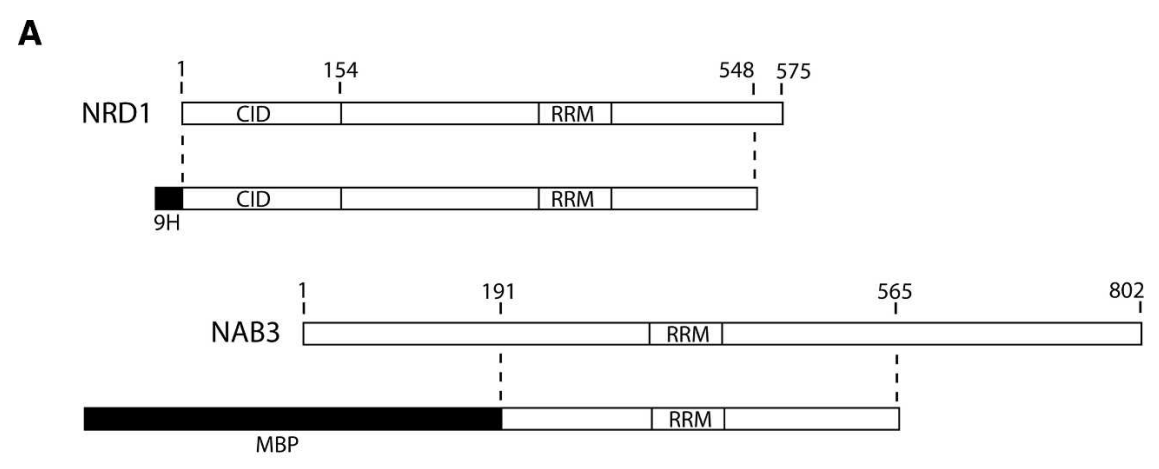

B

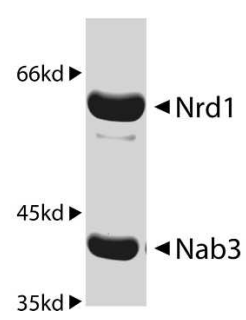

FIGURE 1. Expression and purification of Nrd1-Nab3 heterodimer. $(A)$ Schematic of the regions of Nrd1 and Nab3 included in the expression vector. Nrd1 was His ${ }_{9}$ tagged at the $\mathrm{N}$ terminus and Nab3 was MBP tagged at the $\mathrm{N}$ terminus. (B) SDS-PAGE of purified protein. 
TABLE 1. Physical properties of Nrd1-Nab3 and its complexes with RNA

\begin{tabular}{|c|c|c|c|c|c|c|}
\hline Sample & $\begin{array}{l}M(1-\nu \rho) \\
\quad(\mathrm{kDa})\end{array}$ & $\begin{array}{l}M_{\text {experimental }} \\
\quad(\mathrm{kDa})\end{array}$ & $\begin{array}{c}M_{\text {calc }}(\mathrm{kDa}) \\
\text { for } 1: 1 \text { complexes }\end{array}$ & Stoichiometry $(n)$ & $\begin{array}{c}s_{20, w} \\
\left(10^{-13} \mathrm{~S}\right)\end{array}$ & $f / f_{o}$ \\
\hline Nrd1-Nab3 & $27.4 \pm 1.5^{\mathrm{a}}$ & $102 \pm 6$ & 101.216 & 1:1 Nrd1:Nab3 & $4.3 \pm 0.1$ & 1.9 \\
\hline Nrd1-Nab3 + 11 nt RNA & $29.2 \pm 1.6^{\mathrm{b}}$ & $106 \pm 6$ & 104.652 & $1: 1$ protein:RNA & $4.9 \pm 0.05$ & 1.8 \\
\hline Nrd1-Nab3 + 11 nt RNA & $29.2 \pm 0.7^{c}$ & $106 \pm 3$ & - & - & - & - \\
\hline Nrd1-Nab3 + 35 nt RNA & $66.7 \pm 5.3^{d}$ & $229 \pm 18$ & 112.248 & 2:1 protein:RNA ${ }^{\mathrm{e}}$ & $8.1 \pm 0.1$ & 1.7 \\
\hline
\end{tabular}

${ }^{a}$ Sedimentation equilibrium data were collected at 6, 8, 10, 12, and $14 \mathrm{krpm}$ and $280 \mathrm{~nm}$. Loading concentrations corresponded to 3.1, 6.1, and $9.2 \mu \mathrm{M} \mathrm{Nrd1}-\mathrm{Nab} 3$ and data were analyzed globally in terms of a single ideal solute. Excellent fits were observed.

${ }^{b}$ Sedimentation equilibrium data were collected at 6, 8, 10, and $12 \mathrm{krpm}$ and $280 \mathrm{~nm}$. Loading concentrations corresponded to 1.4, 2.2, 4.5, and $6.7 \mu \mathrm{M}$ of Nrd1-Nab3 and 1.05 equivalents of 11-nt RNA. Data were analyzed globally in terms of a single ideal solute, and excellent data fits were observed. An RNA partial specific volume of $0.50 \mathrm{~cm}^{3} / \mathrm{g}$ was assumed for the calculation of the experimental mass.

${ }^{\mathrm{c} S}$ Sedimentation equilibrium data were collected at 6, 8, 10, and $12 \mathrm{krpm}$ and $290 \mathrm{~nm}$. Loading concentrations corresponded to $20 \mu \mathrm{M}$ Nrd1-Nab3 and $21 \mu \mathrm{M} 11$-nt RNA. Data were analyzed globally in terms of a single ideal solute, and excellent data fits were observed.

${ }^{\mathrm{d} S e d i m e n t a t i o n}$ equilibrium data were collected at 6, 8, and $10 \mathrm{krpm}$ and $280 \mathrm{~nm}$ at a loading concentration of $2.3 \mu \mathrm{M} \mathrm{Nrd1-Nab3}$ and $1.0 \mu \mathrm{M} 35-n t$ RNA. Data were consistent with the presence of two species, and an analysis in terms of two noninteracting species indicates that free $\mathrm{Nrd} 1-\mathrm{Nab} 3$ is present. Data were therefore modeled in terms of free protein $(M[1-\nu \rho]=27.44 \mathrm{kDa})$ and a second species. Excellent data fits were observed. Data were also collected at higher loading concentrations of 4.6 and $6.9 \mu \mathrm{M} \mathrm{Nrd1-Nab3,} \mathrm{with} \mathrm{an} \mathrm{identical} \mathrm{RNA} \mathrm{loading}$ ratio. Even though identical results were obtained at these loading concentrations, the sedimentation equilibrium profiles were consistent with the loss of material and a much higher proportion of free protein heterodimer.

'Based on the error of the method and the sample loading concentrations, we conclude that the data are consistent with a 2:1 Nrd1-Nab3:RNA stoichiometry.

the molecule if it were a sphere. Frictional ratios of $1.0-1.3$ are usually observed for spherical molecules (Tanford 1961), and the value of 1.9 calculated for the Nrd1-Nab3 heterodimer (Table 1) suggests that this protein complex adopts an extended conformation in solution.

\section{Nrd1-Nab3 heterodimer binds to specific RNA sequences in vitro and in vivo}

To assess the ability of the Nrd1-Nab3 complex to bind RNA containing its respective binding motifs, we carried out in vitro filter-binding experiments with purified Nrd1Nab3 and end-labeled RNAs (see Materials and Methods). To examine binding in a simple case we first used RNA oligonucleotides corresponding to $\mathrm{U}^{*} \mathrm{R}^{*}$, the artificial sequence element shown to interact with the Nrd1 protein (Steinmetz and Brow 1996). This 43-nucleotide (nt) RNA contains a single consensus Nrd1 site and a single consensus Nab3 site (Fig. 2A). (Throughout the article, a Nrd1 site will be designated by NR and a Nab3 site by NA.)

Figure $2 \mathrm{~B}$ shows the binding curve for $\mathrm{U}^{*} \mathrm{R}^{\star} \mathrm{RNA}$. The fraction of bound RNA increases as the Nrd1-Nab3 protein concentration is increased from 0 to $5 \mu \mathrm{M}$. The concentration of Nrd1-Nab3 at which $50 \%$ of the target RNA is bound is $\sim 2.75 \mu \mathrm{M}$. Binding affinity determined by these filter-binding experiments is a relative affinity, however, due to the presence of nonspecific competitor RNA (see Materials and Methods).

To confirm that the binding specificity of Nrd1-Nab3 for the $\mathrm{U}^{*} \mathrm{R}^{*} \mathrm{RNA}$ is due to the presence of the Nrd1 and Nab3 motifs, mutant RNAs that contain changes in the Nrd1 site (NR), the Nab3 site (NA), or both sites (NA-NR) were synthesized (Fig. 2A). These single-site mutations were previously identified as point-mutant suppressors of NRD1-dependent down-regulation of a selectable marker gene in vivo (Steinmetz and Brow 1996). Mutation of these sites also reduced binding by the $\mathrm{Nrd} 1$ protein in vitro (Steinmetz and Brow 1998). As expected, mutations in the Nrd1 or Nab3 motifs decrease the binding affinity of the Nrd1-Nab3 heterodimer (Fig. 2B). The Nab3 site mutation appears to be more deleterious to binding affinity than the Nrd1 site mutation, although the endpoint of this binding curve suggests that a substantial fraction of this particular RNA is unavailable for binding. In this case the binding curves may be interpreted as indicating equivalent binding of the two mutants. A combination of the two motif mutations almost abolishes binding to the RNA, signifying that the Nrd1 and Nab3 sites are the primary determinants of specific binding to this RNA.

To extend our analysis to a naturally occurring Nrd1Nab3 termination element, we carried out filter-binding experiments on the snR13 terminator. Via a mutational strategy, we previously showed the importance of the $\mathrm{Nrd} 1$ and Nab3 sites to the function of this terminator (Carroll et al. 2004). The corresponding 35-nt RNA we use in this study contains two Nrd1 sites and two Nab3 sites (Fig. 2C) and includes the region downstream of SNR13 most critical for termination (Carroll et al. 2004). Figure 2D shows the binding curve for this interaction. The snR13 binding curve exhibits a marked contrast to that of the $\mathrm{U}^{*} \mathrm{R}^{\star}$ binding curve in both shape and binding affinity. The concentration of Nrd1-Nab3 at which $50 \%$ of the target RNA is bound is $\sim 500 \mathrm{nM}$. The fraction of bound RNA changes over a very narrow concentration range of Nrd1-Nab3, suggesting possible cooperative binding. 
To examine the relative importance of the four Nrd1and Nab3-binding sites in the snR13 RNA, single-site mutations were also tested in the filter-binding assay (Fig. 2C). These base changes were previously identified in a mutational screen for nonpoly(A) termination elements downstream of the SNR13 gene, and they represent the most frequent base changes that reduced the efficiency of the snR13 terminator (Carroll et al. 2004). All four single-site mutations bind less well to the Nrd1-Nab3 heterodimer than the wild-type (WT) snR13 RNA, though they affect the relative binding affinity to varying degrees (Fig. 2D). The NA2 RNA exhibits a binding curve most similar to that of the snR13 WT RNA with just a slight decrease in binding affinity. The NR1 and the NR2 RNAs have nearly identical binding curves and more of a decrease in binding affinity. The NA1 RNA shows the weakest binding to the Nrd1-Nab3 heterodimer of the single-site mutations tested. As in $\mathrm{U}^{*} \mathrm{R}^{*}$, the most important single binding motif is a Nab3 site.

Single-site mutations, however, only result in minor differences to the relative binding affinity of the snR13 RNA. The most deleterious single mutation, NA1, affects the affinity by a factor of 5 . These binding studies are consistent with the in vivo effects of snR13 terminator single-site mutations, in which single motif mutations do not have a prominent effect on termination efficiency (Carroll et al. 2004). Due to the minor effect of single motif mutations, four double motif mutations were also
A GGGAAAACGAAAUAAAUCUCUUUGUAAAACGGUUCAUCCUUAU WT GGGAAAACGAAAUAAAUCUCUU UGAAAACGGUUCAUCCUUAU NR GGGAAAACGAAAUAAAUCUCGUUGUAAAACGGUUCAUCCUUAU NA GGGAAAACGAAAUAAAUCUC UUGCAAAAGGUUCAUCCUUAU NA-NR

B

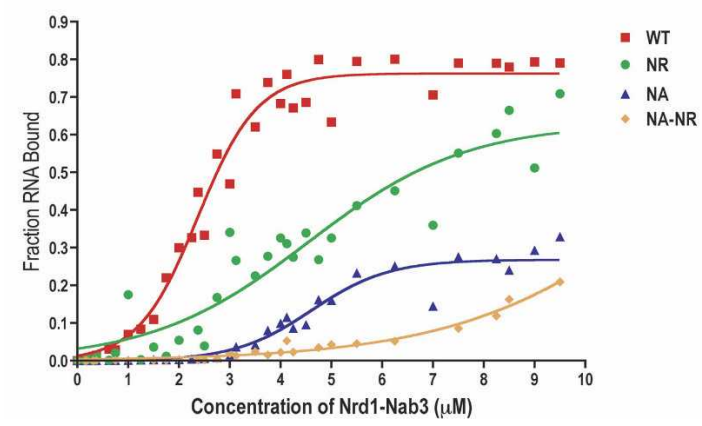

C $\begin{array}{llll}\text { CNrd1 Nab3 Nrd1 Nab3 } & \\ \text { CCCCGUAGAAAAUCUUAGUAACCUUCUUACAUUG } & \text { WT } \\ \text { CCCCGAAGAAAUCUUAGUAAUCCUUCUUACAUUG } & \text { NR1 } \\ \text { CCCCGUAGAAAAUCUGAGUAAUCCUUCUUACAUUG } & \text { NA1 } \\ \text { CCCCGUAGAAAAUCUUAGGAAUCCUUCUUACAUUG } & \text { NR2 } \\ \text { CCCCGUAGAAAAUCUUAGUAAUCCUUAUUACAUUG } & \text { NA2 } \\ \text { CCCCGAAGAAAAUCUGAGUAAUCCUUCUUACAUUG } & \text { NR1-NA1 } \\ \text { CCCCGUAGAAAAUCUUAGGAAUCCUUAUUACAUUG } & \text { NR2-NA2 } \\ \text { CCCCGUAGAAAAUCUGAGGAAUCCUUCUUACAUUG } & \text { NA1-NR2 } \\ \text { CCCCGAAGAAAUCUUAGUAAUCCUUAUUACAUUG } & \text { NR1-NA2 }\end{array}$

D

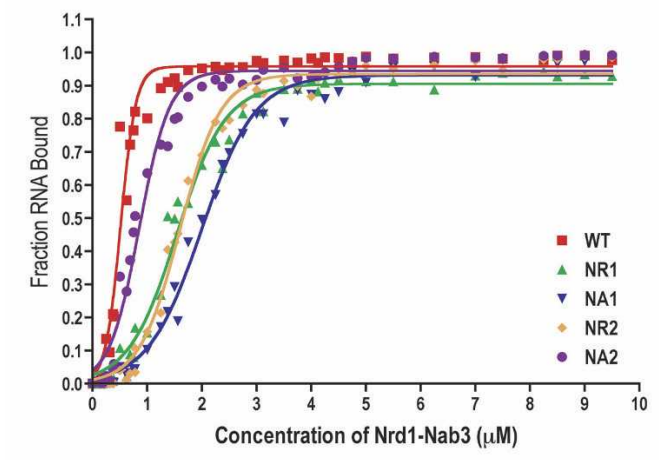

E

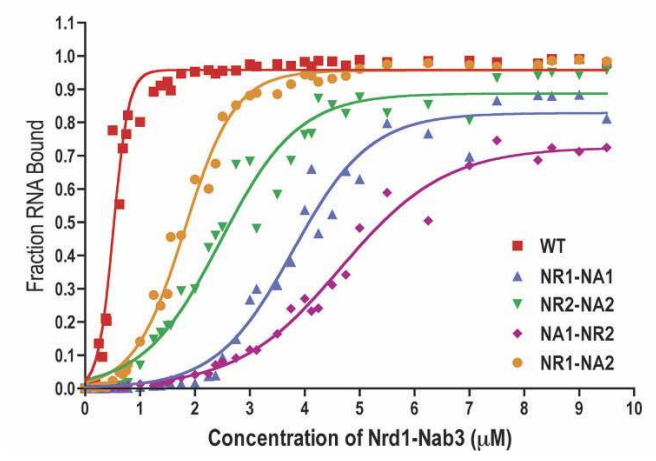

FIGURE 2. Binding affinity and specificity of Nrd1-Nab3 heterodimer. (A) Sequence of U6R ${ }^{\star}$ RNA oligos. (B) Binding curves of U6R $R^{\star}$ RNA oligos to Nrd1-Nab3. (C) Sequence of snR13 RNA oligos. (D) Binding curves of WT and single mutant snR13 RNA oligos to Nrd1-Nab3. (E) Binding curves of WT and double mutant snR13 RNA oligos to Nrd1-Nab3. Nrd1 (NR) and Nab3 (NA) motifs are underlined in red. Single base changes in the binding motifs are highlighted. Filter binding data were fit to a four parameter logistic equation using GraphPad Prism software. 
tested via filter binding. These RNA oligonucleotides contain mutations in both an Nrd1 site and an Nab3 site (Fig. 2C). Predictably, the four double mutant RNAs exhibit even poorer binding affinity than the single-site mutations (Fig. 2E). The NA1-NR2 RNA is the poorest substrate in this series for binding to the Nrd1-Nab3 heterodimer, resulting in a 10 -fold decrease in binding affinity.

To correlate in vitro binding with in vivo termination efficiency, an identical 35-nt snR13 DNA fragment was cloned into an $A D H$-GFP vector (Fig. 3A; Carroll et al. 2004). The plasmid was transformed into yeast, and total RNA from the resultant strain was analyzed by Northern blot with a probe to GFP. The 35-nt fragment derives from a larger 108-nt sequence previously shown to confer Nrd1dependent termination (Steinmetz et al. 2001). In the $A D H$-GFP vector, the 35-nt sequence exhibits a low level of GFP expression similar to that of the larger 108-nt sequence (Fig. 3B, cf. lanes 1 and 3). Thus, sequences sufficient for termination are contained within the smaller 35-nt fragment.

The three most deleterious double mutant variants of the 35-nt snR13 sequence tested in the in vitro binding

A

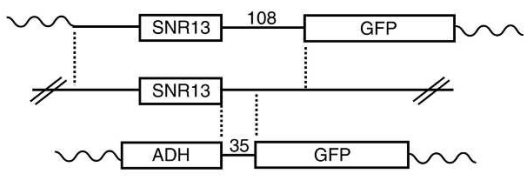

B

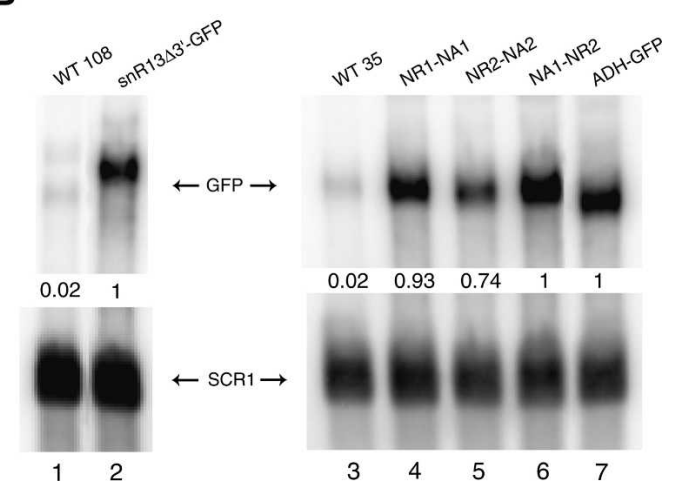

FIGURE 3. Termination efficiency of snR $13 \mathrm{WT}$ and double mutant variants. (A) Schematic of GFP readthrough vectors. (Middle) The SNR13 gene in its genomic locus. (Top) A plasmid containing the SNR13 promoter, gene, and 108 base pairs (bp) of downstream sequence. (Bottom) A plasmid containing $35 \mathrm{bp}$ of snR13 downstream sequence driven by the $A D H$ promoter. Vertical dashed lines indicate regions of identical sequence. Wavy lines indicate the vector. (B) Northern blot of total RNA from yeast harboring the GFP readthrough plasmids. GFP signal was corrected with loading control to SCR1 and is an average of two experiments. Lanes 2 and 7 contain vectors with no snR13 downstream sequences and represent maximum transcription readthrough. These maxima were set to 1 and all other lanes were expressed as a fraction of that maximum. experiments were also cloned into the $A D H$-GFP vector. The Nrd1 and Nab3 motif mutations compromise the function of the snR13 terminator and result in increased GFP readthrough (Fig. 3B, lanes 4, 5, and 6). The measured in vivo termination efficiency correlates with the in vitro binding shown in Figure 2E. The NR2-NA2 mutation is the least severe of this set with $\sim 74 \%$ GFP readthrough and $\sim 2.5$-fold reduction in binding affinity. The NA1-NR2 mutation is most severe in both cases with $\sim 100 \%$ GFP readthrough and $\sim 10$-fold reduction in binding affinity. Thus, in vitro effects mirror in vivo effects: mutations in both Nrd1 and Nab3 motifs result in a significant reduction of Nrd1-Nab3 binding and subsequent termination efficiency. We note that the central Nrd1 and Nab3 sites in the snR13 terminator are the most important elements, and like the sites in the $\mathrm{U}^{*} \mathrm{R}^{\star}$ sequence are separated by a single base.

\section{Electrophoretic separation of Nrd1-Nab3-RNA complexes}

To visualize complex formation between the Nrd1-Nab3 protein and labeled RNA, we carried out electrophoretic mobility shift assays (EMSA). The WT U6R* RNA, containing one Nrd1 site and one Nab3 site, shows a shifted band that is fairly constant in mobility as the protein concentration is increased (Fig. 4A). The three $\mathrm{U}^{*} \mathrm{R}^{*}$ mutant RNAs (NA, NR, NA-NR) display a decreased amount of shifted band with equivalent amounts of slower mobility complex in NA and NR. This correlates with the decreased binding affinity of Nrd1-Nab3 for these RNAs as determined by the filter-binding experiments.

Gel shifts with the panel of snR13 RNAs (Fig. 4B) also support the filter-binding results. Poorer binding affinity is reflected in the appearance of less of the slower mobility band. In contrast to $\mathrm{U}_{6} \mathrm{R}^{\star}$, as the $\mathrm{Nrd} 1-\mathrm{Nab} 3$ protein concentration is increased, the WT snR13 RNA complexes migrate more slowly. This possible higher-order complex formation is preserved in the NR1, NA2, and NR1-NA2 mutant RNAs. However, the remaining five snR13 mutants tested maintain a fairly consistent mobility of the shifted species (NA1, NR1-NA1, NR2, NR2-NA2, NA1-NR2). The NA1-NR2 shifted band in particular seems to maintain the same mobility as the protein concentration is increased.

Figure 4C shows a gel shift with the panel of snR13 RNAs at $5 \mu \mathrm{M}$ Nrd1-Nab3 on the same gel to highlight the difference in mobility of the shifted species. The correlation of weak binding with the most rapidly migrating shifted species is consistent with binding of a single heterodimer to these RNAs. In contrast, the WT and less severe mutant RNAs appear to bind multiple heterodimers, resulting in a slower mobility complex. The failure to observe numerous discrete bands with slower mobility, indicative of multiple heterodimers, may be due to the extended structure of the Nrd1-Nab3 heterodimer as determined from centrifugation. 

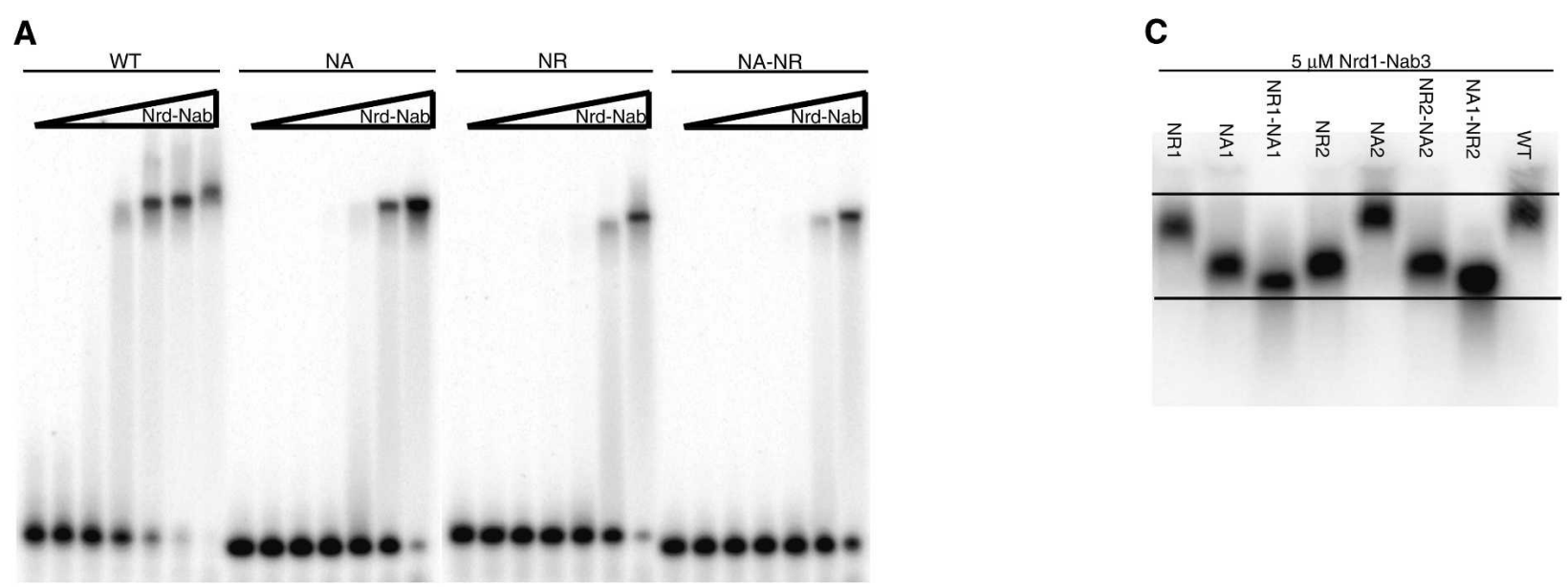

B

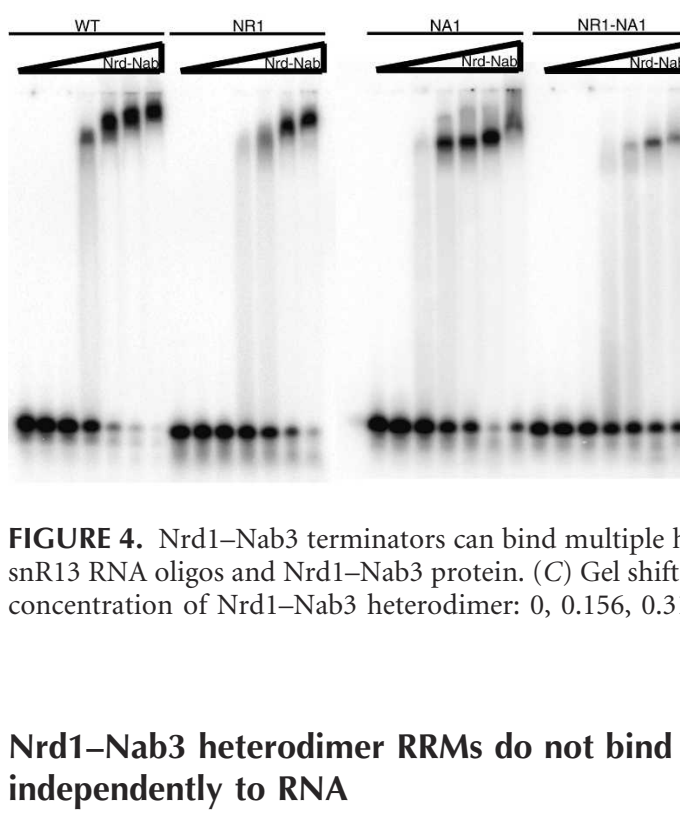

Footprint assays were also performed to probe RNAprotein interactions. Figure $5 \mathrm{~A}$ shows the footprint of Nrd1-Nab3 on U6R* RNA following RNase A digestion. On U6R* WT RNA, the footprint extends over the Nab3and Nrd1-binding motifs, as well as toward the $5^{\prime}$ end of the RNA. In the NA mutant RNA, we see a loss of protection over both the Nab3- and Nrd1-binding motifs. This suggests that the binding of the two RRMs in a single heterodimer is not independent and indicates that interaction with both sites is required for optimal binding.

Figure $5 \mathrm{~B}$ shows the footprint of Nrd1-Nab3 on snR13 RNA following RNase $T_{1}$ digestion. As the protein concentration is increased, the area of protection on the WT RNA is shown to extend over both Nrd1 sites. In the NR1 mutant RNA, we see loss of protection on the corresponding first Nrd1 motif (NR1), but maintenance of protection on the second Nrd1 motif (NR2). This protection may be to a slightly lesser degree than in the WT case. In the NA1 mutant RNA, there is a loss of protection on the corresponding Nab3 site (NA1), as well as on the second Nrd1 site (NR2) directly adjacent to this first Nab3 motif. This suggests that one heterodimer binds NA1 and NR2. These two motifs are separated by a single base as in the case for $\mathrm{U}^{*} \mathrm{R}^{\star}$. In addition, we observe a slight loss of protection on the first Nrd1 motif (NR1), $5^{\prime}$ to the mutated Nab3 site. Similar to $\mathrm{U}^{*} \mathrm{R}^{\star}$, a mutation in a Nab3 site affects protection at an Nrd1 site. Taken together, these results indicate that closely spaced Nrd1 and Nab3 motifs are protected by the same heterodimer.

\section{Hydrodynamic analysis of Nrd1-Nab3-RNA complexes}

In order to determine the stoichiometry of RNA-protein complexes, a series of sedimentation equilibrium and velocity experiments was carried out on a 1:1.05 molar ratio of the Nrd1-Nab3 heterodimer and an 11-nt RNA (see Materials and Methods) containing the central Nab3and Nrd1-binding motifs (NA1 and NR2) of the snR13 
A

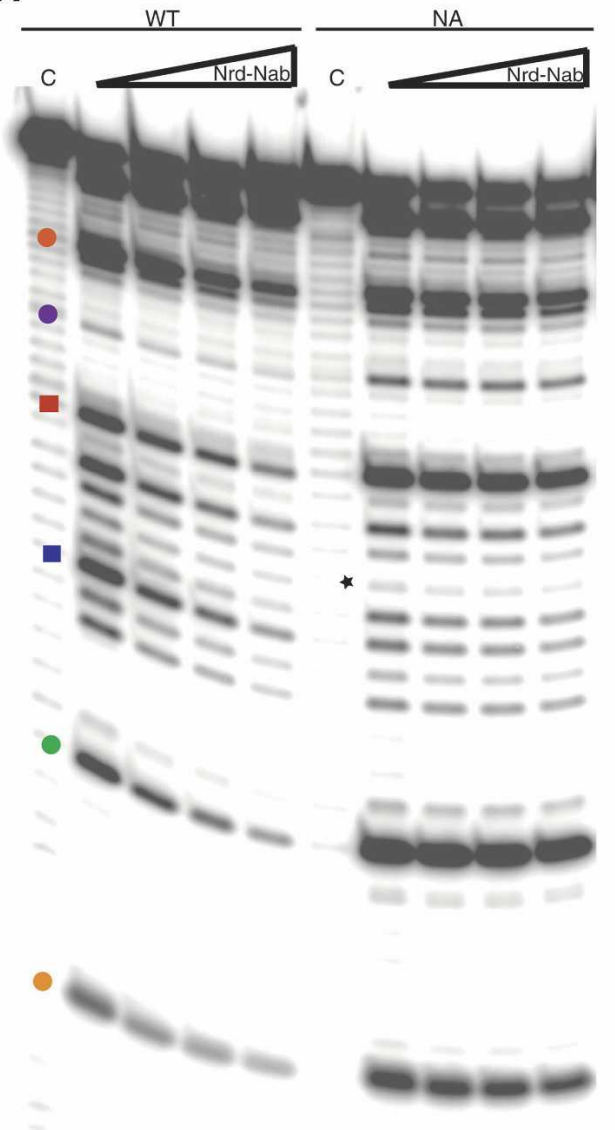

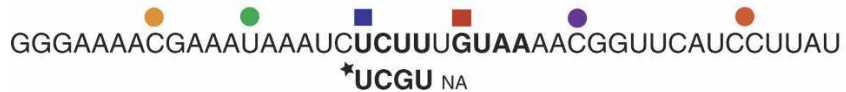

B

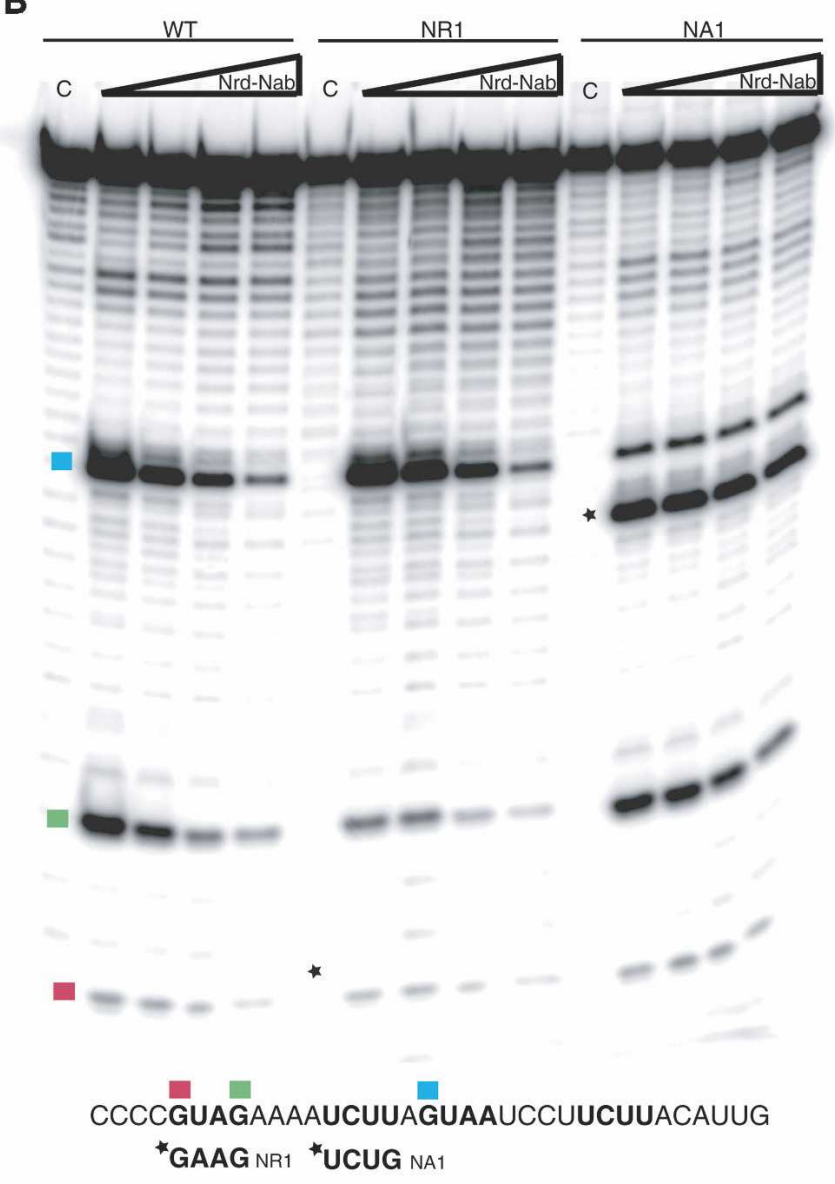

FIGURE 5. Footprint of Nrd1-Nab3 on RNA. (A) RNase A footprinting of U6R ${ }^{\star}$ RNA oligos and Nrd1-Nab3. RNase A cuts after cytosine and uridine residues in single-stranded RNA. The WT U6R* sequence is indicated at the bottom with the NA mutant RNA sequence change underneath. (B) RNase T1 footprinting of snR13 RNA oligos and Nrd1-Nab3. RNase T1 cuts after guanosine residues in single-stranded RNA. The WT snR13 sequence is indicated at the bottom with the NR1 and NA1 mutant RNA sequence changes underneath. The "C" indicates a control lane containing no RNase. Wedge represents increasing concentration of Nrd1-Nab3 heterodimer: 0, 0.625, 1.25, and 2.5 $\mu \mathrm{M}$. Mutations are indicated by an asterisk. Colored squares represent bases in an Nrd1 or a Nab3 motif and colored circles represent additional bases along the RNA. Locations on the RNA oligo and corresponding position on the gel are denoted by the squares and circles.

terminator. Sedimentation equilibrium experiments carried out at rotor speeds of 6-12 krpm and Nrd1-Nab3 loading concentrations of $1.4,2.2,4.5$, and $6.7 \mu \mathrm{M}$ are consistent with the presence of a single species. A global analysis in terms of a single ideal solute returns a molecular mass of $106 \pm 6 \mathrm{kDa}$ (Table 1; data not shown). Even though this value is essentially identical, within the error of the method, to that determined for the Nrd1-Nab3 heterodimer, it is reproducibly larger and consistent with a 1:1 protein:RNA stoichiometry.

Sedimentation velocity experiments carried out at a loading concentration of $6.7 \mu \mathrm{M}$ clearly demonstrate that the species is not Nrd1-Nab3 alone (Fig. 6B) and are consistent with a single species having a measured $s_{20, \mathrm{w}}$ of $4.9 \pm 0.05 \mathrm{~S}$ (Table 1). At higher concentration of the
Nrd1-Nab3-11-nt RNA complex there is evidence of association (data not shown) that is not apparent at the same concentration of protein alone. Together, these experiments demonstrate that the Nrd1-Nab3 heterodimer forms a 1:1 complex with the 11-nt RNA and that this complex retains the overall asymmetry of the protein based on the calculated frictional ratio of 1.8 (Table 1), indicating an extended conformation.

In order to characterize the complex formed between Nrd1-Nab3 and the 35-nt snR13 RNA, a series of sedimentation velocity experiments was carried out at different protein to RNA ratios. Experiments conducted at low protein to RNA ratios reveal the presence of a prominent 5.6 S species (Fig. 6C-E). At the lowest protein to RNA ratios we also see some evidence for free RNA. As the 


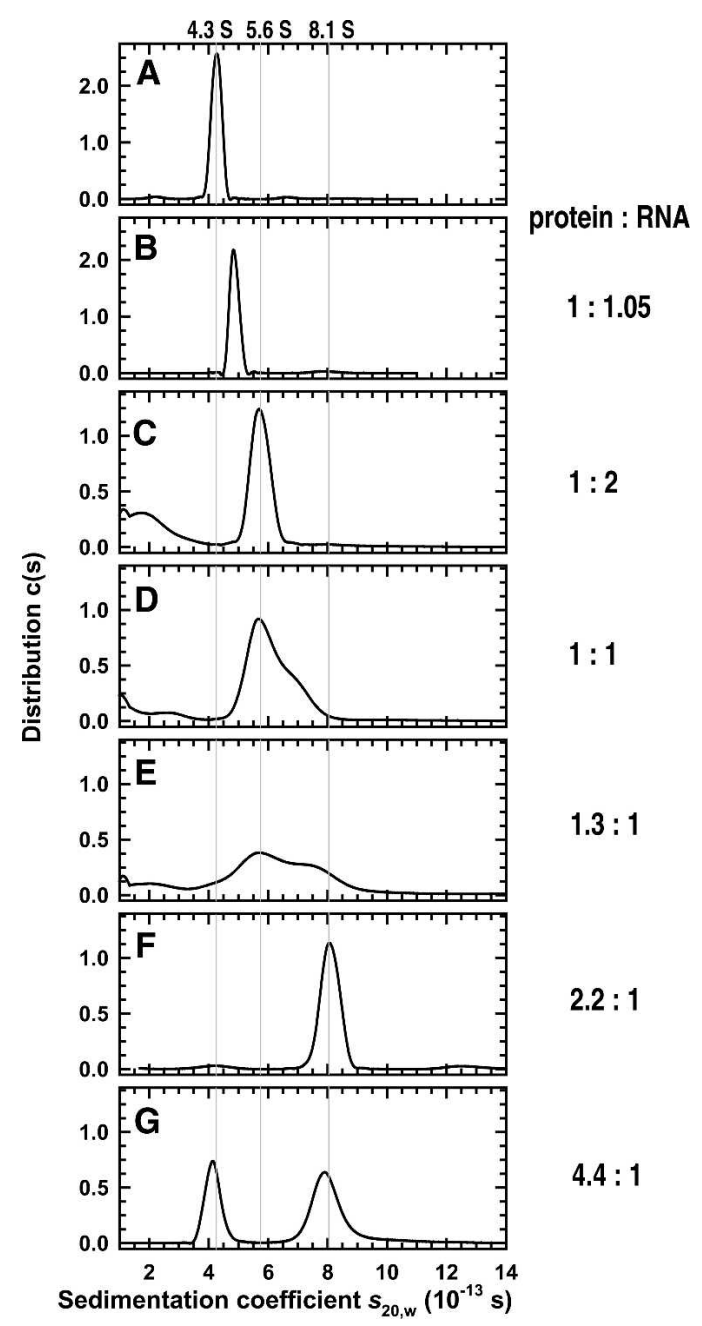

FIGURE 6. Sedimentation velocity experiments on Nrd1-Nab3 and its complexes with RNA. $\mathrm{c}(s)$ distributions based on sedimentation velocity data collected at $55 \mathrm{krpm}, 280 \mathrm{~nm}$, and $4.0^{\circ} \mathrm{C}$ are shown. (A) The distribution for Nrd1-Nab3 at a loading concentration of $10 \mu \mathrm{M}$. (B) The distributions for a 1:1.05 Nrd1-Nab3 to snR13 11-nt RNA mixture at $6.7 \mu \mathrm{M}$. Data for the complex at $6.7 \mu \mathrm{M}$ can be modeled in terms of a single species with an $\mathrm{s}_{20, \mathrm{w}}$ of $4.9 \mathrm{~S}$. The $\mathrm{c}(s)$ distributions for Nrd1-Nab3 and snR13 35 nt RNA at nominal loading concentrations of $(C)$ 1:2 (2.75 $\mu \mathrm{M}$ Nrd1-Nab3), (D) 1:1 (5.5 $\mu \mathrm{M}$ Nrd1-Nab3), (E) 1.3:1 (5.5 $\mu \mathrm{M}$ Nrd1-Nab3), (F) 2.2:1 (7.1 $\mu \mathrm{M}$ Nrd1-Nab3), and $(G)$ 4.4:1 (7.4 $\mu \mathrm{M}$ Nrd1-Nab3) protein: RNA. Note the progressive transition from a 1:1 Nrd1-Nab3 to RNA $5.6 \mathrm{~S}$ complex, to a $2: 1 \mathrm{Nrd} 1-\mathrm{Nab} 3$ to RNA $8.1 \mathrm{~S}$ complex. Excess RNA for the 1:2, 1:1, and 1.3:1 mixtures is noted at sedimentation values $<4$. The gray lines highlight the sedimentation coefficients of the free Nrd1-Nab3, the 1:1 complex with snR13 35-nt RNA, and the 2:1 complex with the same RNA.

protein to RNA ratio is increased we see evidence for a larger complex (Fig. 6E-G). At a 2.2:1 loading ratio, all of the protein is complexed, and data analysis in terms of a single species returns a sedimentation coefficient of $8.1 \pm$ $0.1 \mathrm{~S}$ (Fig. 6F; Table 1). At a 4.4:1 loading ratio of protein to RNA we observe the presence of more than one species, and the distribution of $S$ values is consistent with the presence of a free Nrd1-Nab3 heterodimer and an $\sim 8 \mathrm{~S}$ complex (Fig. 6G).

The value of the sedimentation coefficient suggests that more than one Nrd1-Nab3 heterodimer is present in the $8 \mathrm{~S}$ complex, an observation confirmed by sedimentation equilibrium experiments (data not shown). In these experiments a 2.3:1 protein:RNA loading ratio was used at protein concentrations of $2.3,4.6$, and $6.9 \mu \mathrm{M}$. Data collected at 6, 8, and $10 \mathrm{krpm}$ were consistent with the presence of two species, and an analysis in terms of two noninteracting species indicated that free Nrd1-Nab3 was present. Accordingly, data were modeled in terms of the presence of free protein and a second species, and in all cases excellent fits were obtained. Even though all data sets returned similar buoyant molecular masses for the complex, it was noted that the proportion of free protein increased with the increasing loading concentration. This is likely due to the formation of higher-order RNA-protein complexes. Furthermore, at 4.6 and $6.9 \mu \mathrm{M}$ protein, sample losses were apparent as the rotor speed was incrementally increased. Therefore, only the lowest concentration data were considered (data not shown). The buoyant molecular mass of $66.7 \pm 5.3 \mathrm{kDa}$ demonstrates the dimerization of Nrd1-Nab3 heterodimers, and based on this the complex contains $2 \pm 1$ snR13 RNA species (Table 1 ). This value is slightly higher than the RNA content predicted by sedimentation velocity. These observations are consistent, within the error of the method, with the sedimentation velocity titrations that support a 2:1 Nrd1-Nab3:RNA stoichiometry (Fig. 6). The $8 \mathrm{~S}$ complex is further characterized by a frictional ratio of 1.7 (Table 1), showing that it is elongated.

\section{DISCUSSION}

The Nrd1-Nab3 complex plays several roles in gene regulation by directing termination of snRNA and snoRNA transcripts (Steinmetz et al. 2001), some mRNA transcripts (Arigo et al. 2006a; Houalla et al. 2006), and a class of intergenic and anti-sense transcripts (Arigo et al. 2006b; Lykke-Andersen and Jensen 2006; Thiebaut et al. 2006). In addition, the Nrd1-Nab3 complex facilitates RNA degradation by directing transcripts to the nuclear exosome (Vasiljeva and Buratowski 2006). The first step in all of these functional roles is the recognition of specific RNA sequences in nascent Pol II transcripts.

\section{Specificity of Nrd1-Nab3 RNA binding}

Steinmetz and Brow (1996, 1998) identified sequences within the artificial $\mathrm{U}^{*} \mathrm{R}^{\star}$ terminator that are important for binding Nrd1 and downregulating expression of a selectable marker gene. Overexpression of the RNA-binding protein Nab3 suppresses nrd1 temperature-sensitive mutations, and Nab3 forms a complex with Nrd1 in vivo 
(Conrad et al. 2000). With the discovery of natural Nrd1Nab3 targets it became apparent that Nrd1- and Nab3binding sites are important for snRNA and snoRNA termination (Steinmetz et al. 2001). In subsequent work we used an in vivo genetic selection approach to more precisely define sequences required for Nrd1- and Nab3directed termination at several yeast snoRNA terminators. From libraries of mutant terminators we identified mutations that reduce nonpoly $(\mathrm{A})$ termination efficiency. This selection identified the consensus sequences GUA[A/G] and UCUU as the most frequently mutated targets (Carroll et al. 2004). Both of these sequence motifs are also present in the $\mathrm{U}^{*} \mathrm{R}^{\star}$ terminator and other suspected nonpoly $(\mathrm{A})$ terminators (Steinmetz et al. 2001; Morlando et al. 2002).

Using independently expressed Nrd1 and Nab3 RRM domains we showed that Nrd1 preferentially interacts with the GUAA motifs in the snR47 terminator, while the Nab3 RRM binds preferentially to a UCUU-containing RNA also derived from SNR47 (Carroll et al. 2004). More recently we have identified a CUT terminator that consists only of Nab3 (UCUU) binding sites (Arigo et al. 2006b). This terminator does not function efficiently in an nab3-11 mutant strain but does function in an nrd1-102 mutant strain containing a ts mutation in the Nrd1 RRM. For this terminator, interaction of the Nab3 RRM with UCUU sequences is sufficient for termination. Nrd1 RRM function is not required in this case but a mutation in the CTDinteraction domain $(n r d 1-101)$ reduces termination efficiency, indicating that $\mathrm{Nrd} 1$ is still required for termination even in the absence of RNA interaction. Presumably, Nrd1 acts in this case to physically link Nab3 to the transcriptional machinery. Taken together these results indicate that both Nrd1 and Nab3 function to direct nonpoly(A) termination.

In this article we have extended in vitro binding studies to look at Nrd1 and Nab3 in the more natural context of the heterodimer, as they most likely exist in vivo (Conrad et al. 2000). We performed binding assays on the same $\mathrm{U}_{6} \mathrm{R}^{*} \mathrm{RNA}$ used in previous studies and on the SNR13 $\operatorname{nonpoly}(\mathrm{A})$ terminator. Mutation of either the Nrd1- or Nab3-binding motif in $\mathrm{U}^{*} \mathrm{R}^{\star}$ reduces binding, with the Nab3 mutant having a more severe effect. Footprint experiments on the Nab3 motif mutant RNA exhibit a concomitant loss of protection at the Nrd1 motif, indicating that the two RRMs do not bind independently. The 35- nt snR13 RNA contains two Nrd1-binding and two Nab3binding motifs. Single motif mutations reduce binding but only by a factor of $2-5$, while mutating one Nrd1 and one Nab3 site leads to as much as a 10-fold reduction in binding and a corresponding loss of termination efficiency in vivo. Taken together, the data presented here indicate that GUA[A/G] and UCUU sequences are necessary for Nrd1-Nab3 binding and termination.

Are Nrd1- and Nab3-binding sites sufficient to direct nonpoly(A) termination? The 35-nt snR13 terminator binds Nrd1-Nab3 and directs efficient termination in a reporter assay (Fig. 3). Recent work has suggested that the snR13 and snR65 terminators also contain sequences recognized by the cleavage and polyadenylation machinery (Steinmetz et al. 2006). However, mutations in these poly(A) signals, although contributing to a readthrough phenotype when the terminator is located in an intron, have not been shown to cause readthrough on their own but only in combination with other upstream mutations, often in a GUA[A/G] or UCUU motif (Steinmetz et al. 2006). The 35-nt snR13 terminator described in this work does not contain AU-rich cleavage/polyadenylation signals, arguing that cleavage/polyadenylation signals are not necessary for nonpoly $(\mathrm{A})$ termination. Furthermore, cleavage/polyadenylation signals have been shown to disrupt the production of mature and functional snoRNAs (Fatica et al. 2000). Thus, the primary and significant determinant of snoRNA nonpoly(A) termination is the Nrd1-Nab3 pathway and any involvement by the cleavage/poly(A) pathway seems to be a secondary "fail-safe" pathway.

\section{Affinity of Nrd1-Nab3 RNA binding}

Previous studies of Nrd1 and Nab3 interaction with RNA have employed isolated RRM domains, and the results of these studies have yielded inconsistent results. The Nrd1 RRM has been shown to interact with the $\mathrm{U}^{*} \mathrm{R}^{\star}$ sequence with a $K_{d}$ of $\sim 10 \mathrm{nM}$ (Steinmetz and Brow 1998). Using a similar Nrd1 RRM expression construct we previously examined the binding of the Nrd1 RRM to RNA sequences derived from the snR47 terminator (Carroll et al. 2004). While an accurate binding constant was not determined, the apparent $K_{d}$ of Nrd1 RRM binding to a GUAAcontaining RNA derived from the snR47 terminator was $\sim 1 \mu \mathrm{M}$, several orders of magnitude weaker than reported for $\mathrm{U}^{*} \mathrm{R}^{\star}$. We observed a similar affinity $(\sim 0.3 \mu \mathrm{M})$ for binding of the Nab3 RRM to a UCUU-containing RNA derived from the snR47 terminator (Carroll et al. 2004). These binding affinities to the snR47 elements are more consistent with the binding by single RRMs derived from proteins containing multiple RRMs (Maris et al. 2005).

The reported affinity $(\sim 10 \mathrm{nM})$ of the Nrd1 RRM for $\mathrm{U} \mathrm{R}^{\star}$ is among the strongest reported interactions of a single RRM with RNA. U1A binds to its target sequence, a single-stranded loop, with subnanomolar affinity when the $\mathrm{U} 1$ hairpin forms but with about $10^{3}$ lower affinity to the same sequence in a single-stranded RNA (Law et al. 2006). Since the $\mathrm{U}^{*} \mathrm{R}^{\star}$ sequence is not predicted to form a stable hairpin, it seems unlikely that this is the basis for highaffinity interaction. The RRM of the human splicing regulator FOX1 also binds its target with nanomolar efficiency through specific interactions of an aromatic amino acid just C-terminal to the RNP1 motif (Auweter et al. 2006). These additional interactions extend the 
RNA-binding surface, leading to enhanced affinity and specificity. Whether Nrd1 can make similar contacts is not clear. The consensus binding site for Nrd1 is less than the eight bases recognized by FOX1, and the position adjacent to the RNP1 sequence is not occupied by an aromatic amino acid in Nrd1. Perhaps in the Nrd1-Nab3 heterodimer the RRMs are held in a conformation that reduces binding affinity. Understanding why the $\mathrm{Nrd} 1$ RRM alone binds so tightly to $\mathrm{U}^{*} \mathrm{R}^{\star}$ whereas the Nrd1Nab3 heterodimer does not will require further structural studies.

To examine binding of the Nrd1-Nab3 heterodimer to a natural nonpoly $(\mathrm{A})$ terminator, we studied the snoRNA terminator derived from the SNR13 gene. In this case the apparent $K_{d}$ is $\sim 500 \mathrm{nM}$, and we provide evidence that multiple heterodimers bind in a cooperative manner. The apparent affinity of snR13 binding is also weaker than reported for the Nrd1 RRM binding to $\mathrm{U}^{*} \mathrm{R}^{\star}$ but is similar to the affinity we observe for the heterodimer binding to $\mathrm{U}_{6} \mathrm{R}^{*}$. The actual binding of the Nrd1-Nab3 heterodimer is likely to be tighter, however, because we have used poly(U) as a nonspecific competitor in order to observe discrete bands in EMSA experiments. Similar experiments (data not shown) in the absence of poly(U) indicate a $K_{d}$ of $\sim 300 \mathrm{nM}$. This affinity is consistent with reported binding constants of other RRM-containing proteins with their natural RNA targets (Maris et al. 2005), and the presence of competitor RNA in our studies represents a realistic environment in which Nrd1-Nab3 must find its target in the nuclear milieu.

\section{Cooperative binding of the Nrd1-Nab3 heterodimer to the snR13 terminator}

Nrd1-Nab3 binding to the 35-nt snR13 RNA displays a steep binding curve. This observation, together with gel shift and centrifugation data showing the binding of multiple heterodimers, suggests that Nrd1-Nab3 binds cooperatively to terminators with multiple binding sites. To explain the results of experiments showing multiple binding events, we propose the model shown in Figure 7. The model describes how RNA fragments with more than one set of Nrd1-Nab3-binding sites might associate with the Nrd1-Nab3 heterodimer to form higher-order complexes. $\mathrm{U}^{*} \mathrm{R}^{\star}$ has only a single pair of sites and is not likely to bind more than one heterodimer (Fig. 7A). The 11-nt central part of the snR13 terminator also binds a single heterodimer (Fig. 7B). In contrast, the model shows how two heterodimers can bind to the 35-nt snR13 RNA (Fig. 7C). Gel shift experiments on the snR13 RNAs indicate the formation of higher-order complexes and support a model of multiple binding events.

The formation of complexes with more than one heterodimer is also seen by centrifugation. Sedimentation equilibrium and velocity experiments have shown that
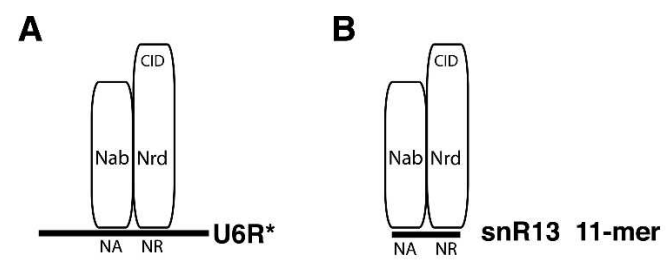

C
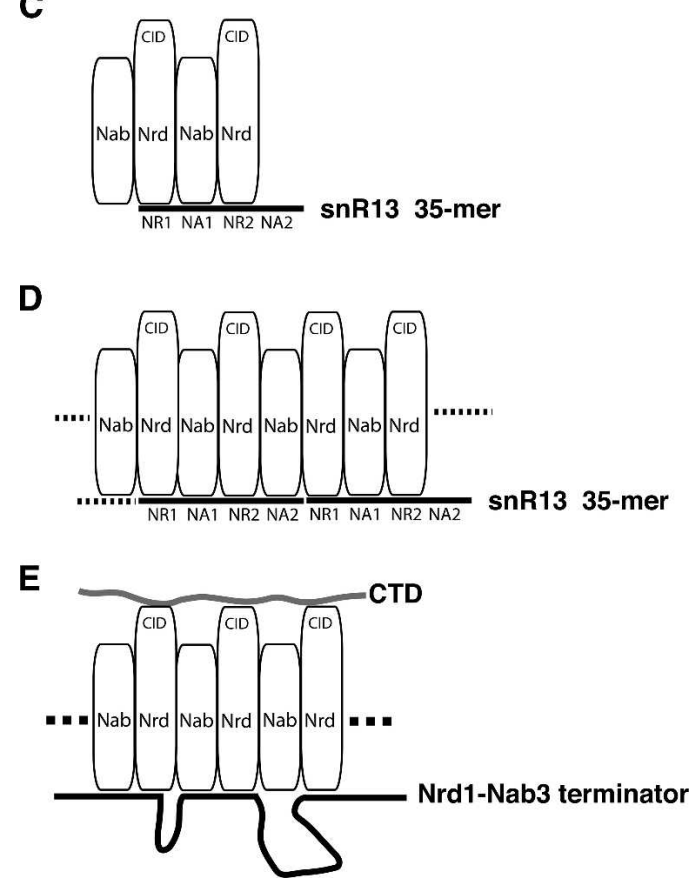

FIGURE 7. Model of Nrd1-Nab3 heterodimer binding to Nrd1-Nab3 RNA terminators. (A) The U6R ${ }^{*}$ RNA contains one Nab3 (NA) and one $\mathrm{Nrd1}$ (NR) site and allows for the binding of one Nrd1-Nab3 heterodimer. (B) The snR13 11-nt RNA also binds one Nrd1-Nab3 heterodimer. $(C)$ The snR13 35-nt RNA contains four potential binding sites. The first heterodimer may bind to the central NA1-NR2 site, allowing for the binding of a second heterodimer to the NR1 site or to the NA2 site (not shown). (D) The unoccupied Nab3 side of the heterodimer would then be free to bind the NA2 site of another RNA, thus seeding the formation of larger protein-RNA complexes. (E) Model for Nrd1-Nab3 binding to multiple sites within an mRNA and subsequent interaction of numerous Nrd1 CIDs with multiple CTD repeats.

Nrd1-Nab3 is a monodisperse heterodimer that does not self-associate to form higher-order complexes. This heterodimer is elongated in shape and forms a 1:1 complex with a short 11-nt RNA containing single GUAA Nrd1-binding and UCUU Nab3-binding motifs. The complex formed retains the asymmetry of the parent protein and shows some self-association that is not apparent with the unbound heterodimer. In contrast, the complex formed with a 35-nt snR13 RNA containing two Nrd1-binding and two Nab3binding motifs has a 2:1 stoichiometry (Fig. 7C), demonstrating that the RNA plays a role in oligomerization of the Nrd1-Nab3 heterodimer. The shape of the complex retains the overall asymmetry noted for the free Nrd1-Nab3 heterodimer and its 1:1 complex with the shorter RNA. 
Given the similarity of the central Nrd1- and Nab3binding sites to the $\mathrm{U}^{*} \mathrm{R}^{\star}$ sequence we propose that these sites (snR13 NA1 and NR2) bind the first heterodimer. Footprinting results and the mild effect of the NR1-NA2 mutant in filter-binding experiments are consistent with this proposal. A second heterodimer may bind to either side. Given the more pronounced effect of mutations in NR1, we propose that this is the preferred secondary site. Once this second heterodimer binds, the unoccupied Nab3 RRM would be available to bind additional RNA molecules. In this fashion large complexes could be formed on a given RNA (Fig. 7D). Higher-order multimers could explain the loss of sample at high protein concentration during centrifugation experiments. This cooperative binding model also helps explain the relatively minor effects of single mutations on snoRNA terminators (Carroll et al. 2004) and the presence of multiple Nrd1- and Nab3-binding sites in several snoRNA terminators. In addition, we have recently shown that multiple mutations in Nrd1- and Nab3-binding sites in the NRD1 mRNA are required to abrogate Nrd1-mediated autoregulation (Arigo et al. 2006a). Figure 7E shows how multiple sites within an mRNA may interact with oligomerized Nrd1-Nab3.

\section{Nonpoly(A) termination}

Why is termination triggered through binding to multiple relatively weak binding sites? First, this form of binding allows more flexibility in placement of key cis elements. Second, the presence of redundant sequences insures that single mutations will not disrupt the termination pathway. In support of the potential utility of such a system, the yeast poly(A) signals are less well conserved than their mammalian counterparts. The efficiency and positioning elements are both degenerate and redundant (Zhao et al. 1999) insuring that transcription termination does not read through into closely adjacent genes. Third, the cooperative nature of the binding insures that inadvertent sequences resembling the nonpoly $(\mathrm{A})$ terminator will not accidentally lead to premature termination. Fourth, regulating the availability of the binding proteins over a narrow concentration range can directly impact termination efficiency. In conclusion, it seems that a collection of low-affinity Nrd1and Nab3-binding sites comprises a functional snoRNA terminator.

The oligomerization aspect of Nrd1-Nab3 binding also affords an interesting possibility regarding the termination mechanism. Previously, it was shown that CTD truncations are synthetically lethal with $n r d 1$ ts alleles (Conrad et al. 2000). As Nrd1-Nab3 heterodimer binds its respective sites in the emerging RNA transcript, the CIDs of Nrd1 available for binding to the CTD repeats accumulate (Fig. 7E). Perhaps when some critical number of Nrd1 CIDs bind to the CTD this imparts a termination signal to the polymerase. Such a termination function could be analogous to that of the $\operatorname{poly}(\mathrm{A})$ termination factor Pcf11, which has a CID related to that of Nrd1. Pcf11 associates with both the Pol II CTD and RNA and has been shown to disrupt paused ternary elongation complexes (Zhang et al. 2005). It is possible that the Nrd1-Nab3 complex plays a similar role in nonpoly $(\mathrm{A})$ termination.

To fully understand the nonpoly(A) termination mechanism, more work will obviously be needed to define optimal Nrd1- and Nab3-binding sites and their preferred contexts and spacing. In particular, it will be important to study Nrd1 and Nab3 interactions in larger complexes containing the nuclear exosome. The present article provides important data on the affinity, specificity, and cooperativity of Nrd1-Nab3 heterodimer binding to RNA. These results show that this RNA interaction is the key to recognition of nonpoly(A) terminators.

\section{MATERIALS AND METHODS}

\section{Expression and purification of Nrd1-Nab3 heterodimer}

Because expression of $\mathrm{Nrd} 1$ and Nab3 individually resulted in low yields of unstable protein, we coexpressed these RNA-binding proteins from vector pST39 (Tan 2001). To assist purification, we His, tagged the $\mathrm{N}$ terminus of $\mathrm{Nrd} 1$ and MBP tagged the $\mathrm{N}$ terminus of Nab3. The Nrd1-Nab3 heterodimer was expressed in E. coli strain BL21 grown in $\mathrm{L}$ broth at $37^{\circ} \mathrm{C}$ to an $\mathrm{OD}_{600}$ of 1 and induced with IPTG at $15^{\circ} \mathrm{C}$ overnight. Cells were broken by sonication, clarified by centrifugation, and the fusion proteins were purified by metal affinity chromatography. After proteolytic removal of the tags with TEV, the protein was desalted into buffer containing $0.15 \mathrm{M} \mathrm{NaCl}, 50 \mathrm{mM}$ MES (pH 6.0), 10\% glycerol, 0.1 mM EDTA, and $0.5 \mathrm{mM}$ DTT, and further purified by chromatography on heparin-agarose and Resource $\mathrm{S}$. The final protein shown in Figure 1B contains two polypeptides with the expected sizes. Protein concentrations were determined by spectroscopy using extinction coefficients $(280 \mathrm{~nm})$ derived by summing the contributions of tyrosine and tryptophan residues (Pace et al. 1995).

\section{Sample preparation for centrifugation}

All samples were prepared by diluting stock solutions of an 11-nt (AUCUUAGUAAU) snR13 RNA $\left(\mathrm{A}_{260}=27.2,236 \mu \mathrm{M}\right)$, the 35-nt snR13 (Fig. 2C) RNA $\left(\mathrm{A}_{260}=240,716 \mu \mathrm{M}\right)$, or the coexpressed Nrd1-Nab3 heterodimer $\left(A_{280}=10.0\right.$ or 17.1) into $150 \mathrm{mM} \mathrm{NaCl}, 20$ $\mathrm{mM}$ MES (pH 6.8), 5\% (v/v) glycerol, and $5 \mathrm{mM}$ 2-mercaptoethanol. Protein stocks were in $150 \mathrm{mM} \mathrm{NaCl}, 20 \mathrm{mM}$ MES (pH 6.8), and $10 \%(\mathrm{v} / \mathrm{v})$ glycerol, whereas RNA stocks were in water.

\section{Sedimentation equilibrium}

Sedimentation equilibrium experiments were conducted at $4.0^{\circ} \mathrm{C}$ on a Beckman Optima XL-A analytical ultracentrifuge. Samples (loading volume of $130 \mu \mathrm{L}$ ) of Nrd1-Nab3, 1:1.05 mixture of Nrd1-Nab3 and 11-nt snR13 RNA, and a 2.3:1 mixture of Nrd1Nab3 and 35-nt snR13 RNA were studied at various loading 
concentrations and rotor speeds (6-14 krpm). Data were acquired as an average of six absorbance measurements at $280 \mathrm{~nm}$ or $290 \mathrm{~nm}$, depending on the loading concentration, using a radial spacing of $0.001 \mathrm{~cm}$. Sedimentation equilibrium was achieved within $48 \mathrm{~h}$. Data were analyzed globally in terms of various species analysis models using SEDPHAT 4.0 (Schuck 2003, 2006b, and references therein) to obtain the buoyant molecular mass $M(1-v \rho)$. Solution densities $(\rho)$ and partial specific volumes $(\nu)$ were used to determine the molecular mass $(M)$. Solution densities were measured at $20.00^{\circ} \mathrm{C}$ on a Mettler-Toledo DE51 density meter and corrected to values for $\rho$ at $4.0^{\circ} \mathrm{C}$. The value of $v$ for Nrd1-Nab3 was calculated using the amino acid composition in SEDNTERP (Hayes et al. 2006), whereas a value of 0.50 $\mathrm{cm}^{3} / \mathrm{g}$ was assumed for the RNA (Durschlag 1986). In all cases excellent data fits were observed. Effective partial specific volumes for nucleic acids range from 0.45 to $0.55 \mathrm{~cm}^{3} / \mathrm{g}$ and are very dependent on the base composition, ionic strength, solution $\mathrm{pH}$, buffering agent, and the overall hydration of the polymer (Durschlag 1986; Eisenberg 1989; Woodward and Lebowitz 1980). Using the observed 1:1 protein:11-nt RNA stoichiometry, the experimentally determined buoyant molecular masses of Nrd1$\mathrm{Nab} 3$ and its complex return a partial specific volume of $0.48 \mathrm{~cm}^{3} /$ $\mathrm{g}$ for the RNA in the complex. This value is within the range expected for nucleic acids.

\section{Sedimentation velocity}

Sedimentation velocity experiments were conducted at $4.0^{\circ} \mathrm{C}$ on a Beckman Optima XL-A analytical ultracentrifuge. Samples of Nrd1-Nab3, 1:1.05 mixture of Nrd1-Nab3 and 11-nt snR13 RNA, various mixtures of Nrd1-Nab3 and 35-nt snR13 RNA (loading volume of $300 \mu \mathrm{L}$ ) were studied at a loading absorbance of $\sim 1.0$ and rotor speeds of $55 \mathrm{krpm}$. Aluminum double centerpiece cells were used. One hundred to 125 scans were acquired at $\sim 3$-min intervals as a single absorbance measurement at 280, 290, or $294 \mathrm{~nm}$ and a radial spacing of $0.003 \mathrm{~cm}$. Experiments were carried out in duplicate and data were analyzed in terms of a single species, as well as a $\mathrm{c}(\mathrm{s})$ distribution using SEDFIT 9.2 (Schuck 2000, 2006a, and references therein). Sedimentation coefficients $(s)$ were corrected to $s_{20, w}$ based on the solvent density and viscosity $(\eta)$ calculated based on the composition in SEDNTERP (Hayes et al. 2006). Excellent data fits were observed for all the continuous c(s) analyses.

\section{Hydrodynamic calculations}

The sedimentation coefficients $s$ obtained were used to calculate the frictional coefficient $f$, using the relation

$$
s=\mathrm{M}(1-v \rho) / \mathrm{N} f
$$

where $M$ is the molecular mass, $v$ is the partial specific volume, $\rho$ is the solution density, and $N$ is Avogadro's number. The value of $f$ was compared to the smallest possible frictional coefficient $f_{o}$ for a sphere of equivalent mass (Tanford 1961):

$$
f_{\mathrm{o}}=6 \pi \eta \mathrm{r}=6 \pi \eta(3 \mathrm{M} \nu / 4 \pi \mathrm{N})^{1 / 3}
$$

where $r$ is the radius of the sphere and $\eta$ is the solution viscosity.

\section{RNA-binding assays}

RNA oligonucleotides were synthesized by Integrated DNA Technologies (IDT) and end labeled with T4 polynucleotide kinase and $\left[\gamma_{-}{ }^{32} \mathrm{P}\right]$ ATP. The labeling reactions were passed over MicroSpin G-50 columns (Amersham Biosciences) to remove unincorporated $\left[\gamma^{32} \mathrm{P}\right]$ ATP. Binding reactions were performed in $20 \mathrm{mM}$ MES (pH 6.8), $40 \mathrm{mM} \mathrm{NaCl}, 1.6 \mathrm{mM} \mathrm{MgCl}_{2}, 0.03 \mathrm{mM}$ EDTA, $0.2 \mathrm{mM}$ DTT, 3\% glycerol, and $0.1 \mathrm{mg} / \mathrm{mL}$ poly(U) containing $\sim 5 \mathrm{nM}$ labeled RNA and varying amounts of Nrd1$\mathrm{Nab} 3$ complex. After incubation of the binding reaction at $25^{\circ} \mathrm{C}$ for sufficient time to reach equilibrium (20 $\mathrm{min}$ ), the percentage of RNA bound was determined by filter binding (Wong and Lohman 1993). Filter-binding data were fit to a four parameter logistic equation (Hill equation) using GraphPad Prism software (v.4.0b).

Binding reactions were also subjected to electrophoretic mobility shift assays (EMSA) (Steinmetz and Brow 1998; Carroll et al. 2004). After the 20-min incubation, sucrose and dye were added to the reactions and they were loaded onto $5 \%$ polyacrylamide gels that had been prerun for $1 \mathrm{~h}$ in a $4^{\circ} \mathrm{C}$ cold room. Gels were run for $5-6 \mathrm{~h}$ at constant temperature $\left(\sim 8^{\circ} \mathrm{C}\right)$ and then exposed in a PhosphorImager cassette.

Nonspecific competitor RNA was included in binding reactions to eliminate aggregation of RNA-protein complexes (data not shown). Increasing levels of poly(U) (Sigma) allowed the formation of discrete Nrd1-Nab3-RNA complexes. It should be noted that $\operatorname{poly}(\mathrm{U})$, even at the high concentrations used in the experiments, has little effect on specific binding. The binding to snR13 RNA is only slightly affected, and the apparent $K_{d}$ of $500 \mathrm{nM}$ with poly(U) is close to the observed $K_{d}$ of $300 \mathrm{nM}$ without poly(U). Thus, poly $(\mathrm{U})$ was included in all filter-binding, EMSA, and footprint experiments for consistency. The presence of the nonspecific competitor means that all binding parameters are relative and likely represent a slight underestimation of actual binding affinities.

\section{RNA footprint assays}

Binding reactions were subjected to either RNase $A$ or $T_{1}$ digestions as described by Ambion. RNA was fractionated on $15 \%$ acrylamide-urea gels prerun for $1 \mathrm{~h}$ at $30 \mathrm{~W}$. Gels were dried and exposed in a PhosphorImager cassette.

\section{In vivo readthrough assay}

The ADH-GFP vector was previously described (Carroll et al. 2004). All plasmids were transformed into yeast strain BY4741 (Brachmann et al. 1998). Total RNA was isolated from yeast strains as previously described (Carroll et al. 2004). Twenty micrograms of RNA were run on a $1.25 \%$ denaturing formaldehyde MOPS agarose and transferred to nitrocellulose using a pressure blotter. Hybridizations were performed at $65^{\circ} \mathrm{C}$ with a GFP riboprobe (transcribed with T3 RNA polymerase from a linearized GFP-containing plasmid) or $42^{\circ} \mathrm{C}$ with an SCR1 probe (random primed with Klenow DNA polymerase on a PCR product containing the entire gene). Blots were scanned on a PhosphorImager and bands were quantified using ImageQuant software.

\section{ACKNOWLEDGMENTS}

This research was supported by National Institutes of Health grant GM66108 (J.L.C.) and the Intramural Research Program of the 
$\mathrm{NIH}$, National Institute of Diabetes and Digestive and Kidney Diseases (R.G.). We thank Drs. Rachel Green, Jon Lorsch, and Cecelia Trainor for a critical reading of the manuscript.

Received October 9, 2006; accepted December 6, 2006.

\section{REFERENCES}

Arigo, J.T., Carroll, K.L., Ames, J.M., and Corden, J.L. 2006a. Regulation of yeast NRD1 expression by premature transcription termination. Mol. Cell 21: 641-651.

Arigo, J.T., Eyler, D.E., Carroll, K.L., and Corden, J.L. 2006b. Termination of cryptic unstable transcripts is directed by yeast RNA-binding proteins Nrd1 and Nab3. Mol. Cell 23: 841-851.

Auweter, S.D., Fasan, R., Reymond, L., Underwood, J.G., Black, D.L., Pitsch, S., and Allain, F.H. 2006. Molecular basis of RNA recognition by the human alternative splicing factor Fox-1. EMBO J. 25: 163-173.

Bentley, D.L. 2005. Rules of engagement: Co-transcriptional recruitment of pre-mRNA processing factors. Curr. Opin. Cell Biol. 17: 251-256.

Brachmann, C.B., Davies, A., Cost, G.J., Caputo, E., Li, J., Hieter, P., and Boeke, J.D. 1998. Designer deletion strains derived from Saccharomyces cerevisiae S288C: A useful set of strains and plasmids for PCR-mediated gene disruption and other applications. Yeast 14: 115-132.

Butler, J.S. 2002. The yin and yang of the exosome. Trends Cell Biol. 12: $90-96$.

Carroll, K.L., Pradhan, D.A., Granek, J.A., Clarke, N.D., and Corden, J.L. 2004. Identification of cis elements directing termination of yeast nonpolyadenylated snoRNA transcripts. Mol. Cell. Biol. 24: 6241-6252.

Conrad, N.K., Wilson, S.M., Steinmetz, E.J., Patturajan, M., Brow, D.A., Swanson, M.S., and Corden, J.L. 2000. A yeast heterogeneous nuclear ribonucleoprotein complex associated with RNA polymerase II. Genetics 154: 557-571.

Durschlag, H. 1986. Specific volumes of biological macromolecules and some other molecules of bilogical interest. In Thermodynamic data for biochemistry and biotechnology (ed. H.J. Hinz), pp. 45-128. Springer-Verlag, Berlin.

Eisenberg, H. 1989. Solution properties of DNA: Sedimentation, scattering of light, $\mathrm{X}$-rays and neutrons, and viscometry. In Landolt-Börnstein New Series Biophysics-Nucleic Acids (ed. W. Saenger), pp. 257-276. Springer-Verlag, Berlin.

Fatica, A., Morlando, M., and Bozzoni, I. 2000. Yeast snoRNA accumulation relies on a cleavage-dependent/polyadenylationindependent 3'-processing apparatus. EMBO J. 19: 6218-6229.

Hayes, D.B., Laue, T., and Philo, J. 2006. John Philo's software home page. http://www.jphilo.mailway.com

Houalla, R., Devaux, F., Fatica, A., Kufel, J., Barrass, D., Torchet, C., and Tollervey, D. 2006. Microarray detection of novel nuclear RNA substrates for the exosome. Yeast 23: 439-454.

Houseley, J., LaCava, J., and Tollervey, D. 2006. RNA-quality control by the exosome. Nat. Rev. Mol. Cell Biol. 7: 529-539.

Law, M.J., Rice, A.J., Lin, P., and Laird-Offringa, I.A. 2006. The role of RNA structure in the interaction of U1A protein with U1 hairpin II RNA. RNA 12: 1168-1178.

Lykke-Andersen, S. and Jensen, T.H. 2006. CUT it out: Silencing of noise in the transcriptome. Nat. Struct. Mol. Biol. 13: 860-861.

Maris, C., Dominguez, C., and Allain, F.H. 2005. The RNA recognition motif, a plastic RNA-binding platform to regulate posttranscriptional gene expression. FEBS J. 272: 2118-2131.
Mitchell, P., Petfalski, E., Shevchenko, A., Mann, M., and Tollervey, D. 1997. The exosome: A conserved eukaryotic RNA processing complex containing multiple $3^{\prime} \rightarrow 5^{\prime}$ exoribonucleases. Cell 91: 457-466.

Moore, M.J. 2005. From birth to death: The complex lives of eukaryotic mRNAs. Science 309: 1514-1518.

Morlando, M., Greco, P., Dichtl, B., Fatica, A., Keller, W., and Bozzoni, I. 2002. Functional analysis of yeast snoRNA and snRNA $3^{\prime}$-end formation mediated by uncoupling of cleavage and polyadenylation. Mol. Cell. Biol. 22: 1379-1389.

Pace, C.N., Vajdos, F., Fee, L., Grimsley, G., and Gray, T. 1995. How to measure and predict the molar absorption coefficient of a protein. Protein Sci. 11: 2411-2423.

Proudfoot, N. and O'Sullivan, J. 2002. Polyadenylation: A tail of two complexes. Curr. Biol. 12: R855-R857.

Schuck, P. 2000. Size distribution analysis of macromolecules by sedimentation velocity ultracentrifugation and Lamm equation modeling. Biophys. J. 78: 1606-1619.

Schuck, P. 2003. On the analysis of protein self-association by sedimentation velocity analytical ultracentrifugation. Anal. Biochem. 320: 104-124.

Schuck, P. 2006a. Sedfit. http://www.analyticalultracentrifugation. com/default.htm

Schuck, P. 2006b. SEDPHAT http://www.analyticalultracentrifugation. com/sedphat/sedphat.htm

Steinmetz, E.J. and Brow, D.A. 1996. Repression of gene expression by an exogenous sequence element acting in concert with a heterogeneous nuclear ribonucleoprotein-like protein, $\mathrm{Nrd1}$, and the putative helicase Sen1. Mol. Cell. Biol. 16: 6993-7003.

Steinmetz, E.J. and Brow, D.A. 1998. Control of pre-mRNA accumulation by the essential yeast protein $\mathrm{Nrd} 1$ requires high-affinity transcript binding and a domain implicated in RNA polymerase II association. Proc. Natl. Acad. Sci. 95: 6699-6704.

Steinmetz, E.J., Conrad, N.K., Brow, D.A., and Corden, J.L. 2001. RNA-binding protein Nrd1 directs poly(A)-independent 3 '-end formation of RNA polymerase II transcripts. Nature 413: 327-331.

Steinmetz, E.J., Ng, S.B., Cloute, J.P., and Brow, D.A. 2006. cis- and trans-Acting determinants of transcription termination by yeast RNA polymerase II. Mol. Cell. Biol. 26: 2688-2696.

Tan, S. 2001. A modular polycistronic expression system for overexpressing protein complexes in Escherichia coli. Protein Expr. Purif. 21: 224-234.

Tanford, C. 1961. Physical chemistry of macromolecules. John Wiley and Sons, Inc., New York.

Thiebaut, M., Kisseleva-Romanova, E., Rougemaille, M., Boulay, J., and Libri, D. 2006. Transcription termination and nuclear degradation of cryptic unstable transcripts: A role for the Nrd1-Nab3 pathway in genome surveillance. Mol. Cell 23: 853-864.

Vasiljeva, L. and Buratowski, S. 2006. Nrd1 interacts with the nuclear exosome for $3^{\prime}$ processing of RNA polymerase II transcripts. Mol. Cell 21: 239-248.

Wong, I. and Lohman, T.M. 1993. A double-filter method for nitrocellulose-filter binding: Application to protein-nucleic acid interactions. Proc. Natl. Acad. Sci. 90: 5428-5432.

Woodward, R.S. and Lebowitz, J. 1980. A revised equation relating DNA buoyant density to guanine plus cytosine content. J. Biochem. Biophys. Methods 2: 307-309.

Zhang, Z., Fu, J., and Gilmour, D.S. 2005. CTD-dependent dismantling of the RNA polymerase II elongation complex by the pre-mRNA $3^{\prime}$ end processing factor, Pcf11. Genes \& Dev. 19: 1572-1580.

Zhao, J., Hyman, L., and Moore, C. 1999. Formation of mRNA $3^{\prime}$ ends in eukaryotes: Mechanism, regulation, and interrelationships with other steps in mRNA synthesis. Microbiol. Mol. Biol. Rev. 63: 405-445. 

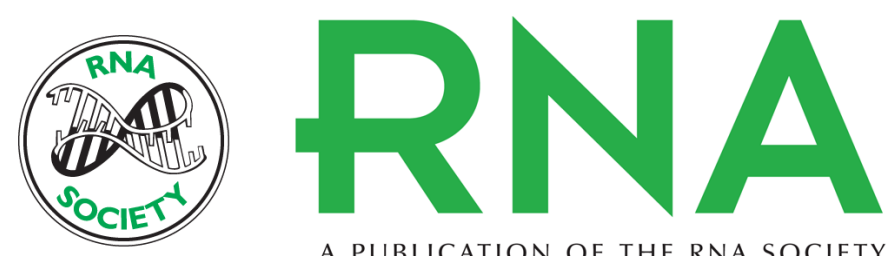

A PUBLICATION OF THE RNA SOCIETY

\section{Interaction of yeast RNA-binding proteins Nrd1 and Nab3 with RNA polymerase II terminator elements}

Kristina L. Carroll, Rodolfo Ghirlando, Jessica M. Ames, et al.

RNA 2007 13: 361-373 originally published online January 19, 2007

Access the most recent version at doi:10.1261/rna.338407

\section{References This article cites 31 articles, 12 of which can be accessed free at: http://rnajournal.cshlp.org/content/13/3/361.full.html\#ref-list-1}

\section{License}
Email Alerting Receive free email alerts when new articles cite this article - sign up in the box at the Service top right corner of the article or click here.

To subscribe to RNA go to:

http://rnajournal.cshlp.org/subscriptions 